\title{
Calculations of Transition Probabilities for Some Excited Levels of Na I
}

\author{
G. ÇELIK* AND Ş. AteŞ \\ Department of Physics, Faculty of Arts and Science, Selçuk University \\ Campus 42075 Konya, Türkiye \\ (Received November 16, 2007; revised version February 13, 2008)

\begin{abstract}
Transition probabilities are calculated for individual and multiplet lines
\end{abstract} \\ between some excited states in neutral sodium by using the weakest bound \\ electron potential model theory. In the determination of parameters re- \\ quired for calculation of transition probabilities, we employed numerical non- \\ -relativistic Hartree-Fock wave functions for expectation values of radii in \\ all levels. The necessary energy values were taken from experimental energy \\ data in the literature. The obtained results were compared with accepted \\ values taken from National Institute of Standards and Technology data and \\ multi-configurational Hartree-Fock results given by Fischer. A good agree- \\ ment was observed in related excited states.
}

PACS numbers: $31 \cdot 10 .+\mathrm{z}$

\section{Introduction}

The alkali atoms have long served as a test system for various theoretical developments aimed at the accurate determination of spectroscopic data such as transition probabilities, oscillator strengths, and lifetimes. In the last four decades, the determination of spectroscopic data for neutral and ionized atomic systems has been an active research area on account of its considerable interest in astrophysics, plasma physics, laser physics, and thermonuclear fusion research. Many of the modern experimental techniques still encounter difficulties in the exact measurement of transition probabilities, oscillator strengths and lifetimes. The results obtained from experimental methods usually have some uncertainties ranging between $\pm 10-30 \%$ arising from the component deviations including the uncertainties of line intensity measurements, uncertainties of the temperature determination and possible influence of self-absorption effects [1]. Moreover, it is seen from the literature that the values measured by different experimental

${ }^{*}$ corresponding author; e-mail: gultekin@selcuk.edu.tr 
methods can yield different results for the same transitions. Many theoretical methods exist for calculation of transition probabilities in atomic or ionic systems such as the Hartree-Fock approximations, configuration interaction methods, R-matrix methods, semi-empirical methods and many-body perturbation theories. The energy level calculations in especially highly lying Rydberg levels in multi-electron atoms or ions are always difficult problems in theoretical studies, because of indistinguishability of equivalent electrons and the necessity of taking many configurations into account [2]. Therefore, many of theoretical methods in the literature have presented spectroscopic data results belonging to low lying levels rather than highly lying levels.

The multi-electron problems can be solved nearly exactly for systems with a few electrons such as helium. More general multi-electron systems cannot be treated with such precision. It is impossible to solve many-electron systems without imposing severe approximations. However, most of the properties found in the one-electron atom can be carried through the many-electron atoms. The alkali atoms are multi-electron systems with a single electron outside closed shells and the simplest systems that offer the possibility of studying core, valence, and valencecore interactions. Therefore, they have been proving ground of truly many-electron methodologies [3]. Recently, Zheng et al. have employed the weakest bound electron potential model (WBEPM) theory for calculations of various physical parameters in both alkali-metal atoms and other many-electron systems [4-10]. They have obtained very satisfactory results with theoretical and experimental results in the literature for calculation of spectroscopic data.

In the present article, we have calculated transition probabilities for both individual and multiplet lines belonging to some excited levels of atomic sodium by using the WBEPM theory. We have employed numerical non-relativistic HartreeFock (NRHF) wave functions and experimental energy values in the determination of relevant parameters required for calculation of transition probabilities.

\section{Theory and calculation procedure}

The WBEPM theory which is presented by Zheng et al. [11, 12] has been used for determination of spectroscopic data such as energy levels, ionization potentials, transition probabilities, oscillator strengths and lifetimes of excited levels in atomic or ionic multi-electron systems. In this theory, electronic radial wave functions are calculated as a function of the Laguerre polynomials in terms of some parameters determined by using the experimental energy data and the expectation values of radii. Then, some physical parameters can be calculated by using these wave functions given by Zheng et al. [5-7, 11, 12]. The WBEPM theory is based on the choice of zero of energy, the dynamic successive ionization and distinguishing the weakest bounded electron from others in a given multi-electron system. By the separation of the electrons in a given system, complex many-electron problem can be simplified as the single-electron problem and so it can be solved easily [5-7]. 
In WBEPM theory, radial wave function obtained by means of the solution of the Schrödinger equation under non-relativistic conditions for weakest bounded electron is given to be [5-7]:

$$
\begin{aligned}
& R_{n l}(r)=\left(\frac{2 Z^{*}}{n^{*}}\right)^{l^{*}+3 / 2}\left[\frac{2 n^{*}}{(n-l-1) !} \Gamma\left(n^{*}+l^{*}+1\right)\right]^{-1 / 2} \\
& \quad \times \exp \left(-\frac{Z^{*} r}{n^{*}}\right) r^{l^{*}} L_{n-l-1}^{2 l^{*}+1}\left(\frac{2 Z^{*} r}{n^{*}}\right),
\end{aligned}
$$

$n^{*}$ and $l^{*}$ parameters have been given to be

$$
n^{*}=n+d \quad \text { and } \quad l^{*}=l+d,
$$

where $Z^{*}, n^{*}$ and $l^{*}$ quantities are defined to be effective nuclear charge, effective principal quantum number, and effective azimuthal quantum number, respectively. According to the WBEPM theory, the weakest bound electron (WBE) moves in the potential field produced by the nucleus and the non-weakest bound electron (NWBE). This potential field can be divided into two parts, one of which is the Coulomb potential. Because of the orbital penetration effect of the WBE, shielding by the NWBE is not complete. Therefore, an effective nuclear charge $Z^{*}$ is used in the Coulomb term of the potential function. The second part is the dipole potential. This dipole moment affects the behavior of the WBE. It would make the principal quantum number $n$ and the angular momentum quantum number $l$ of the WBE replaced by the effective principal quantum number $n^{*}$ and effective angular momentum quantum number $l^{*}$. The introduction of $d$ effectively modifies the integer quantum numbers $n$ and $l$ into non-integers $n^{*}$ and $l^{*}$. These terms differ from the usual core polarization potential which behaves as $\frac{1}{r^{4}}$ asymptotically, in that the effective dipole moment of the core is used as a parameter rather than being derived from the polarizability of the core in the electric field of the WBE $[5-7,11,12]$.

In WBEPM theory, the determination of $Z^{*}, n^{*}$ and $l^{*}$ parameters is sufficient for the calculation of spectroscopic data. These parameters are obtained by solving Eq. (3) and Eq. (4) together

$$
\begin{aligned}
& I=-\varepsilon=\frac{Z^{* 2}}{2 n^{* 2}}, \\
& \langle r\rangle=\frac{3 n^{* 2}-l^{*}\left(l^{*}+1\right)}{2 Z^{*}},
\end{aligned}
$$

where $\varepsilon$ or $I$ is defined to be the ionization energy and $\langle r\rangle$ is expectation value for radius of the WBE. The ionization energies and expectation values for radii of all states must be known for the parameters $Z^{*}, n^{*}, l^{*}$ to be determined. It is well known that some difficulties in obtaining the parameters directly from theory are still present. Therefore, Zheng has suggested that the ionization energy for the WBE is taken from experimental energy value in literature and expectation value of radius of the WBE is obtained from many different theoretical methods. Then, 
radial transition integral or radial matrix element between two different states can be determined easily by using radial wave functions given in Eq. (1).

The total electric dipole transition probability from $\gamma J M$ to all $M^{\prime}$ levels of $\gamma^{\prime} J^{\prime}$ can be determined as [13]:

$$
A\left(\gamma J M \rightarrow \gamma^{\prime} J^{\prime} M^{\prime}\right)=\frac{64 \pi^{4} e^{2} a_{0}^{2}\left(E_{J^{\prime}}-E_{J}\right)^{3}}{3 h\left(2 J^{\prime}+1\right)} S .
$$

Here, $E_{J^{\prime}}-E_{J}$ is the energy difference between relevant levels and $S$ is the electric dipole line strength. Moreover, the numerical coefficients apply for $E_{J^{\prime}}-E_{J}$ in kaysers $\left(\mathrm{cm}^{-1}\right)$ and $S$ line strength is in atomic units of $e^{2} a_{0}^{2}$. Line strength is determined according to the coupling schemes and the transition types in atomic or ionic systems. The text book given by Cowan [13] has presented in detail how the line strength can be calculated according to considered coupling schemes and for different type transitions. The most important quantity for the calculation of $S$ line strength is determination of the radial transition integral or transition matrix elements. In order to solve the radial parts of the Schrödinger equation in multi-electron systems, the several approximations must be imposed. As well known, $L S$ coupling in light atoms is dominant coupling scheme and in this coupling scheme the electric dipole line strength for transitions between two excited levels can be given as [13]:

$$
\begin{aligned}
& \sqrt{S_{L S}} \\
& \quad \equiv\left\langle\left[\left(\ldots \alpha_{1} L_{1}, l_{2}\right) L\left(\ldots S_{1} s_{2}\right) S\right] J|| r_{N}^{(1)}||\left[\left(\ldots \alpha_{1}^{\prime} L_{1}^{\prime}, l_{2}^{\prime}\right) L^{\prime}\left(\ldots S_{1}^{\prime} s_{2}\right) S^{\prime}\right] J^{\prime}\right\rangle \\
& \quad=\delta_{\alpha_{1} L_{1} S_{1}, \alpha_{1}^{\prime} L_{1}^{\prime} S_{1}^{\prime} \delta_{s s^{\prime}}(-1)^{S+J^{\prime}+L_{1}+l_{2}^{\prime}}\left[J, J^{\prime}, L, L^{\prime}\right]^{1 / 2}} \\
& \quad \times\left(\begin{array}{ccc}
L & S & J \\
J^{\prime} & 1 & L^{\prime}
\end{array}\right)\left(\begin{array}{ccc}
L_{1} & l_{2} & L \\
1 & L^{\prime} & l_{2}^{\prime}
\end{array}\right) P_{l_{2} l_{2}^{\prime}}^{(1)} \\
& P_{l_{\mathrm{i}} l_{\mathrm{f}}}^{(1)}=l_{>}\left\langle n_{\mathrm{i}}, l_{\mathrm{i}}\left|r^{4}\right| n_{\mathrm{f}}, l_{\mathrm{f}}\right\rangle=\int_{0}^{\infty} r^{k+2} R_{n_{\mathrm{i}} l_{\mathrm{i}}}(r) R_{n_{\mathrm{f}} l_{\mathrm{f}}}(r) \mathrm{d} r .
\end{aligned}
$$

$P_{l_{\mathrm{i}} l_{\mathrm{f}}}^{(1)}$ quantity in Eq. $(7)$ is known to be radial transition integral or transition matrix element. In this work, we employed the WBEPM theory for $\left\langle n_{\mathrm{i}}, l_{\mathrm{i}}\left|r^{4}\right| n_{\mathrm{f}}, l_{\mathrm{f}}\right\rangle$ matrix element given in Eq. (7). In the WBEPM theory, for a transition from the level $\left(n_{\mathrm{i}}, l_{\mathrm{i}}\right)$ to the level $\left(n_{\mathrm{f}}, l_{\mathrm{f}}\right)$, the expectation value of $r^{k}$ or radial transition integral is given as [5-7]:

$$
\begin{aligned}
& \left\langle n_{\mathrm{i}}, l_{\mathrm{i}}\left|r^{k}\right| n_{\mathrm{f}}, l_{\mathrm{f}}\right\rangle=\int_{0}^{\infty} r^{k+2} R_{n_{\mathrm{i}} l_{\mathrm{i}}}(r) R_{n_{\mathrm{f}} l_{\mathrm{f}}}(r) \mathrm{d} r \\
& =(-1)^{n_{\mathrm{f}}+n_{\mathrm{i}}+l_{\mathrm{f}}+l_{\mathrm{i}}}\left(\frac{2 Z_{\mathrm{f}}^{*}}{n_{\mathrm{f}}^{*}}\right)^{l_{\mathrm{f}}^{*}}\left(\frac{2 Z_{\mathrm{i}}^{*}}{n_{\mathrm{i}}^{*}}\right)^{l_{\mathrm{i}}^{*}}\left(\frac{Z_{\mathrm{f}}^{*}}{n_{\mathrm{f}}^{*}}-\frac{Z_{\mathrm{i}}^{*}}{n_{\mathrm{i}}^{*}}\right)^{-l_{\mathrm{f}}^{*}-l_{\mathrm{i}}^{*}-k-3}
\end{aligned}
$$




$$
\begin{aligned}
& \times\left[\frac{n_{\mathrm{f}}^{* 4} \Gamma\left(n_{\mathrm{f}}^{*}+l_{\mathrm{f}}^{*}+1\right)}{4 Z_{\mathrm{f}}^{* 3}\left(n_{\mathrm{f}}-l_{\mathrm{f}}-1\right) !}\right]^{-1 / 2}\left[\frac{n_{\mathrm{i}}^{* 4} \Gamma\left(n_{\mathrm{i}}^{*}+l_{\mathrm{i}}^{*}+1\right)}{4 Z_{\mathrm{i}}^{* 3}\left(n_{\mathrm{i}}-l_{\mathrm{i}}-1\right) !}\right]^{-1 / 2} \\
& \times \sum_{m_{1}=0}^{n_{\mathrm{f}}-l_{\mathrm{f}}-1} \sum_{m_{2}=0}^{n_{\mathrm{i}}-l_{\mathrm{i}}-1} \frac{(-1)^{m_{2}}}{m_{1} ! m_{2} !}\left(\frac{Z_{\mathrm{f}}^{*}}{n_{\mathrm{f}}^{*}}-\frac{Z_{\mathrm{i}}^{*}}{n_{\mathrm{i}}^{*}}\right)^{m_{1}+m_{2}}\left(\frac{Z_{\mathrm{f}}^{*}}{n_{\mathrm{f}}^{*}}+\frac{Z_{\mathrm{i}}^{*}}{n_{\mathrm{i}}^{*}}\right)^{-m_{1}-m_{2}} \\
& \times \Gamma\left(l_{\mathrm{f}}^{*}+l_{\mathrm{i}}^{*}+m_{1}+m_{2}+k+3\right) \times \sum_{m_{3}=0}^{S}\left(\begin{array}{c}
l_{\mathrm{i}}^{*}-l_{\mathrm{f}}^{*}+k+m_{2}+1 \\
n_{\mathrm{f}}^{*}-l_{\mathrm{f}}^{*}-1-m_{1}-m_{3}
\end{array}\right) \\
& \times\left(\begin{array}{c}
l_{\mathrm{f}}^{*}-l_{\mathrm{i}}^{*}+k+m_{1}+1 \\
n_{\mathrm{i}}^{*}-l_{\mathrm{i}}^{*}-1-m_{2}-m_{3}
\end{array}\right) \\
& \times\left(\begin{array}{c}
l_{\mathrm{i}}^{*}+l_{\mathrm{f}}^{*}+k+m_{1}+m_{2}+m_{3}+2 \\
m_{3}
\end{array}\right),
\end{aligned}
$$

where $S=\min \left\{n_{\mathrm{f}}-l_{\mathrm{f}}-1-m_{1}, n_{\mathrm{i}}-l_{\mathrm{i}}-1-m_{2}\right\}$ and $k>-l_{\mathrm{f}}^{*}-l_{\mathrm{i}}^{*}-3$. The papers given by Zheng et al. have presented much detailed knowledge about WBEPM theory.

\section{Results and conclusions}

The atomic transition probabilities for multiplet and individual lines between some excited states of neutral sodium have been calculated by using the weakest bound electron potential model theory. This theory is an efficient method especially for transitions between excited levels. In the WBEPM theory, reliability of the results depends on $Z^{*}, n^{*}$ and $l^{*}$ parameters. These parameters have been determined by using experimental ionization energy and expectation values of radius. Since experimental ionization energy data are very precise, determination of the expectation values of the radii is much more crucial than energy values. Therefore, in order to calculate expectation values of radii in this study, we have employed numerical NRHF wave functions [14] more sophisticated than the numerical Coulomb approximation (NCA) wave functions [15] which are commonly employed in the WBEPM theory literature. The experimental ionization energy values have been taken from National Institute of Standards and Technology (NIST) [16].

Our transition probability results for multiplet and individual lines are given in Table. The results of transition probability calculated by using the WBEPM theory have been compared with accepted values taken from NIST [17] which contains recommended values for many transitions and multi configurational HartreeFock (MCHF) results given by Fischer [18]. Experimental and theoretical data for comparisons are quite limited for especially highly excited states which are considered in this study. Moreover, some data have not been tested sensitively for both the multiplet and the individual lines especially in transitions belonging to highly excited levels. The accuracy ranges of accepted values taken from NIST are 
TABLE

Atomic transition probabilities and comparison with the accepted values from NIST and MCHF values for sodium $\left(\times 10^{8} \mathrm{~s}^{-1}\right)$.

\begin{tabular}{|c|c|c|c|}
\hline Transitions & $\begin{array}{l}\text { This } \\
\text { work }\end{array}$ & $\begin{array}{c}\text { MCHF } \\
\text { (Ref. [16]) }\end{array}$ & $\begin{array}{l}\text { Acc. val. } \\
\text { (Ref. [15]) }\end{array}$ \\
\hline $1 s^{2} 2 s^{2} 2 p^{6} 3 p^{2} P \rightarrow 1 s^{2} 2 s^{2} 2 p^{6} 4 s^{2} S$ & $2.54 \cdot 10^{-1}$ & $2.61 \cdot 10^{-1}$ & $2.64 \cdot 10^{-1}$ \\
\hline $1 s^{2} 2 s^{2} 2 p^{6} 3 p^{2} P_{3 / 2} \rightarrow 1 s^{2} 2 s^{2} 2 p^{6} 4 s^{2} S_{1 / 2}$ & $1.69 \cdot 10^{-1}$ & $1.71 \cdot 10^{-1}$ & $1.76 \cdot 10^{-1}$ \\
\hline $1 s^{2} 2 s^{2} 2 p^{6} 3 p^{2} P_{1 / 2} \rightarrow 1 s^{2} 2 s^{2} 2 p^{6} 4 s^{2} S_{1 / 2}$ & $8.53 \cdot 10^{-2}$ & $8.73 \cdot 10^{-2}$ & $8.80 \cdot 10^{-2}$ \\
\hline $1 s^{2} 2 s^{2} 2 p^{6} 3 p^{2} P \rightarrow 1 s^{2} 2 s^{2} 2 p^{6} 3 d^{2} D$ & $5.31 \cdot 10^{-1}$ & $5.07 \cdot 10^{-1}$ & $5.14 \cdot 10^{-1}$ \\
\hline $1 s^{2} 2 s^{2} 2 p^{6} 3 p^{2} P_{3 / 2} \rightarrow 1 s^{2} 2 s^{2} 2 p^{6} 3 d^{2} D_{5 / 2}$ & $5.31 \cdot 10^{-1}$ & $5.07 \cdot 10^{-1}$ & $5.14 \cdot 10^{-1}$ \\
\hline $1 s^{2} 2 s^{2} 2 p^{6} 3 p^{2} P_{3 / 2} \rightarrow 1 s^{2} 2 s^{2} 2 p^{6} 3 d^{2} D_{3 / 2}$ & $8.85 \cdot 10^{-2}$ & $8.45 \cdot 10^{-2}$ & $8.57 \cdot 10^{-2}$ \\
\hline $1 s^{2} 2 s^{2} 2 p^{6} 3 p^{2} P_{1 / 2} \rightarrow 1 s^{2} 2 s^{2} 2 p^{6} 3 d^{2} D_{3 / 2}$ & $4.44 \cdot 10^{-1}$ & $4.23 \cdot 10^{-1}$ & $4.29 \cdot 10^{-1}$ \\
\hline $1 s^{2} 2 s^{2} 2 p^{6} 3 d^{2} D \rightarrow 1 s^{2} 2 s^{2} 2 p^{6} 4 p^{2} P$ & $1.53 \cdot 10^{-3}$ & $1.59 \cdot 10^{-3}$ & $1.58 \cdot 10^{-3}$ \\
\hline $1 s^{2} 2 s^{2} 2 p^{6} 3 d^{2} D_{3 / 2} \rightarrow 1 s^{2} 2 s^{2} 2 p^{6} 4 p^{2} P_{3 / 2}$ & $1.54 \cdot 10^{-4}$ & $1.602 \cdot 10^{-4}$ & $1.59 \cdot 10^{-4}$ \\
\hline $1 s^{2} 2 s^{2} 2 p^{6} 3 d^{2} D_{3 / 2} \rightarrow 1 s^{2} 2 s^{2} 2 p^{6} 4 p^{2} P_{1 / 2}$ & $1.51 \cdot 10^{-3}$ & $1.57 \cdot 10^{-3}$ & $1.57 \cdot 10^{-3}$ \\
\hline $1 s^{2} 2 s^{2} 2 p^{6} 3 d^{2} D_{5 / 2} \rightarrow 1 s^{2} 2 s^{2} 2 p^{6} 4 p^{2} P_{3 / 2}$ & $1.38 \cdot 10^{-3}$ & $1.44 \cdot 10^{-3}$ & $1.43 \cdot 10^{-3}$ \\
\hline $1 s^{2} 2 s^{2} 2 p^{6} 3 d^{2} D \rightarrow 1 s^{2} 2 s^{2} 2 p^{6} 4 f^{2} F$ & $1.42 \cdot 10^{-1}$ & $1.40 \cdot 10^{-1}$ & $1.40 \cdot 10^{-1}$ \\
\hline $1 s^{2} 2 s^{2} 2 p^{6} 3 d^{2} D_{3 / 2} \rightarrow 1 s^{2} 2 s^{2} 2 p^{6} 4 f^{2} F_{5 / 2}$ & $1.32 \cdot 10^{-1}$ & $1.31 \cdot 10^{-1}$ & $1.31 \cdot 10^{-1}$ \\
\hline $1 s^{2} 2 s^{2} 2 p^{6} 3 d^{2} D_{5 / 2} \rightarrow 1 s^{2} 2 s^{2} 2 p^{6} 4 f^{2} F_{1 / 2}$ & $1.42 \cdot 10^{-1}$ & $1.40 \cdot 10^{-1}$ & $1.40 \cdot 10^{-1}$ \\
\hline $1 s^{2} 2 s^{2} 2 p^{6} 3 d^{2} D_{5 / 2} \rightarrow 1 s^{2} 2 s^{2} 2 p^{6} 4 f^{2} F_{5 / 2}$ & $9.49 \cdot 10^{-3}$ & $9.35 \cdot 10^{-3}$ & $9.35 \cdot 10^{-3}$ \\
\hline $1 s^{2} 2 s^{2} 2 p^{6} 3 d^{2} D \rightarrow 1 s^{2} 2 s^{2} 2 p^{6} 5 f^{2} F$ & $4.73 \cdot 10^{-2}$ & $4.702 \cdot 10^{-2}$ & $4.70 \cdot 10^{-2}$ \\
\hline $1 s^{2} 2 s^{2} 2 p^{6} 3 d^{2} D_{3 / 2} \rightarrow 1 s^{2} 2 s^{2} 2 p^{6} 5 f^{2} F_{5 / 2}$ & $4.41 \cdot 10^{-2}$ & $4.389 \cdot 10^{-2}$ & $4.38 \cdot 10^{-2}$ \\
\hline $1 s^{2} 2 s^{2} 2 p^{6} 3 d^{2} D_{5 / 2} \rightarrow 1 s^{2} 2 s^{2} 2 p^{6} 5 f^{2} F_{7 / 2}$ & $4.73 \cdot 10^{-2}$ & $4.702 \cdot 10^{-2}$ & $4.70 \cdot 10^{-2}$ \\
\hline $1 s^{2} 2 s^{2} 2 p^{6} 3 d^{2} D_{5 / 2} \rightarrow 1 s^{2} 2 s^{2} 2 p^{6} 5 f^{2} F_{5 / 2}$ & $3.15 \cdot 10^{-3}$ & $3.134 \cdot 10^{-3}$ & $3.13 \cdot 10^{-3}$ \\
\hline $1 s^{2} 2 s^{2} 2 p^{6} 5 s^{2} S \rightarrow 1 s^{2} 2 s^{2} 2 p^{6} 5 p^{2} P$ & $1.63 \cdot 10^{-2}$ & $1.407 \cdot 10^{-2}$ & $1.43 \cdot 10^{-2}$ \\
\hline $1 s^{2} 2 s^{2} 2 p^{6} 5 s^{2} S_{1 / 2} \rightarrow 1 s^{2} 2 s^{2} 2 p^{6} 5 p^{2} P_{3 / 2}$ & $1.63 \cdot 10^{-2}$ & $1.408 \cdot 10^{-2}$ & $1.43 \cdot 10^{-2}$ \\
\hline $1 s^{2} 2 s^{2} 2 p^{6} 5 s^{2} S_{1 / 2} \rightarrow 1 s^{2} 2 s^{2} 2 p^{6} 5 p^{2} P_{1 / 2}$ & $1.63 \cdot 10^{-2}$ & $1.404 \cdot 10^{-2}$ & $1.42 \cdot 10^{-2}$ \\
\hline $1 s^{2} 2 s^{2} 2 p^{6} 4 d^{2} D \rightarrow 1 s^{2} 2 s^{2} 2 p^{6} 5 p^{2} P$ & $6.33 \cdot 10^{-4}$ & $6.98 \cdot 10^{-4}$ & $6.22 \cdot 10^{-4}$ \\
\hline $1 s^{2} 2 s^{2} 2 p^{6} 4 d^{2} D_{5 / 2} \rightarrow 1 s^{2} 2 s^{2} 2 p^{6} 5 p^{2} P_{3 / 2}$ & $5.73 \cdot 10^{-4}$ & $6.308 \cdot 10^{-4}$ & $5.62 \cdot 10^{-4}$ \\
\hline $1 s^{2} 2 s^{2} 2 p^{6} 4 d^{2} D_{3 / 2} \rightarrow 1 s^{2} 2 s^{2} 2 p^{6} 5 p^{2} P_{1 / 2}$ & $6.26 \cdot 10^{-4}$ & $6.92 \cdot 10^{-4}$ & $6.16 \cdot 10^{-4}$ \\
\hline $1 s^{2} 2 s^{2} 2 p^{6} 4 d^{2} D_{3 / 2} \rightarrow 1 s^{2} 2 s^{2} 2 p^{6} 5 p^{2} P_{3 / 2}$ & $6.36 \cdot 10^{-5}$ & $7.028 \cdot 10^{-5}$ & $6.24 \cdot 10^{-5}$ \\
\hline $1 s^{2} 2 s^{2} 2 p^{6} 4 d^{2} D \rightarrow 1 s^{2} 2 s^{2} 2 p^{6} 5 f^{2} F$ & $2.65 \cdot 10^{-2}$ & $2.628 \cdot 10^{-2}$ & $2.59 \cdot 10^{-2}$ \\
\hline $1 s^{2} 2 s^{2} 2 p^{6} 4 d^{2} D_{5 / 2} \rightarrow 1 s^{2} 2 s^{2} 2 p^{6} 5 f^{2} F_{7 / 2}$ & $2.65 \cdot 10^{-2}$ & $2.627 \cdot 10^{-2}$ & $2.59 \cdot 10^{-2}$ \\
\hline $1 s^{2} 2 s^{2} 2 p^{6} 4 d^{2} D_{3 / 2} \rightarrow 1 s^{2} 2 s^{2} 2 p^{6} 5 f^{2} F_{5 / 2}$ & $2.47 \cdot 10^{-2}$ & $2.454 \cdot 10^{-2}$ & $2.42 \cdot 10^{-2}$ \\
\hline $1 s^{2} 2 s^{2} 2 p^{6} 4 d^{2} D_{5 / 2} \rightarrow 1 s^{2} 2 s^{2} 2 p^{6} 5 f^{2} F_{5 / 2}$ & $1.76 \cdot 10^{-3}$ & $1.751 \cdot 10^{-3}$ & $1.73 \cdot 10^{-3}$ \\
\hline $1 s^{2} 2 s^{2} 2 p^{6} 4 f^{2} F \rightarrow 1 s^{2} 2 s^{2} 2 p^{6} 5 d^{2} D$ & $5.26 \cdot 10^{-4}$ & $5.779 \cdot 10^{-4}$ & $5.75 \cdot 10^{-4}$ \\
\hline $1 s^{2} 2 s^{2} 2 p^{6} 4 f^{2} F_{7 / 2} \rightarrow 1 s^{2} 2 s^{2} 2 p^{6} 5 d^{2} D_{5 / 2}$ & $5.01 \cdot 10^{-4}$ & $5.506 \cdot 10^{-4}$ & $5.48 \cdot 10^{-4}$ \\
\hline $1 s^{2} 2 s^{2} 2 p^{6} 4 f^{2} F_{5 / 2} \rightarrow 1 s^{2} 2 s^{2} 2 p^{6} 5 d^{2} D_{3 / 2}$ & $5.26 \cdot 10^{-4}$ & $5.776 \cdot 10^{-4}$ & $5.75 \cdot 10^{-4}$ \\
\hline $1 s^{2} 2 s^{2} 2 p^{6} 4 f^{2} F_{5 / 2} \rightarrow 1 s^{2} 2 s^{2} 2 p^{6} 5 d^{2} D_{5 / 2}$ & $2.53 \cdot 10^{-5}$ & $2.75 \cdot 10^{-5}$ & $2.74 \cdot 10^{-5}$ \\
\hline
\end{tabular}


TABLE (cont.)

\begin{tabular}{|c|c|c|c|}
\hline Tra & $\begin{array}{l}\text { This } \\
\text { work }\end{array}$ & $\begin{array}{c}\text { MCHF } \\
\text { (Ref. [16]) }\end{array}$ & $\begin{array}{c}\text { Acc. val. } \\
\text { (Ref. [15]) }\end{array}$ \\
\hline $1 s^{2} 2 s^{2} 2 p^{6} 4 f^{2} F \rightarrow 1 s^{2} 2 s^{2} 2 p^{6} 6 d^{2} D$ & $20 \cdot 10^{-4}$ & $2.461 \cdot 10^{-4}$ & $2.46 \cdot 10^{-4}$ \\
\hline $1 s^{2} 2 s^{2} 2 p^{6} 4 f^{2} F_{7 / 2} \rightarrow 1 s^{2} 2 s^{2} 2 p^{6} 6 d^{2} D_{5 / 2}$ & $.09 \cdot 10^{-4}$ & $2.345 \cdot 10^{-4}$ & $2.34 \cdot 10^{-4}$ \\
\hline $1 s^{2} 2 s^{2} 2 p^{6} 4 f^{2} F_{5 / 2} \rightarrow 1 s^{2} 2 s^{2} 2 p^{6} 6 d^{2} D_{3 / 2}$ & $2.20 \cdot 10^{-4}$ & $2.460 \cdot 10^{-4}$ & $2.46 \cdot 10^{-4}$ \\
\hline $1 s^{2} 2 s^{2} 2 p^{6} 4 f^{2} F_{5 / 2} \rightarrow 1 s^{2} 2 s^{2} 2 p^{6} 6 d^{2} D_{5 / 2}$ & $1.04 \cdot 10^{-5}$ & $1.171 \cdot 10^{-5}$ & $1.17 \cdot 10^{-5}$ \\
\hline $1 s^{2} 2 s^{2} 2 p^{6} 5 p^{2} P \rightarrow 1 s^{2} 2 s^{2} 2 p^{6} 6 s^{2} S$ & $1.57 \cdot 10^{-2}$ & $1.604 \cdot 10^{-2}$ & $1.61 \cdot 10^{-2}$ \\
\hline $1 s^{2} 2 s^{2} 2 p^{6} 5 p^{2} P_{3 / 2} \rightarrow 1 s^{2} 2 s^{2} 2 p^{6} 6 s^{2} S_{1 / 2}$ & $.04 \cdot 10^{-2}$ & $1.068 \cdot 10^{-2}$ & $1.07 \cdot 10^{-2}$ \\
\hline $1 s^{2} 2 s^{2} 2 p^{6} 5 p^{2} P_{1 / 2} \rightarrow 1 s^{2} 2 s^{2} 2 p^{6} 6 s^{2} S_{1 / 2}$ & $5.26 \cdot 10^{-3}$ & $5.358 \cdot 10^{-3}$ & $5.36 \cdot 10^{-3}$ \\
\hline $1 s^{2} 2 s^{2} 2 p^{6} 5 d^{2} D$ & $2.63 \cdot 10$ & 2.59 & 2.5 \\
\hline $1 s^{2} 2 s^{2} 2 p^{6} 5 d^{2} D_{5 / 2}$ & $2.38 \cdot 10^{-4}$ & $2.351 \cdot 10^{-4}$ & $2.28 \cdot 10^{-4}$ \\
\hline $1 s^{2} 2 s^{2} 2 p^{6} 5 d^{2} D_{3 / 2} \rightarrow$ & $2.61 \cdot 10^{-4}$ & $2.572 \cdot 10^{-4}$ & $2.50 \cdot 10^{-4}$ \\
\hline $1 s^{2} 2 s^{2} 2 p^{6} 5 d^{2} D_{3}$ & $65 \cdot 10^{-5}$ & $2.613 \cdot 10^{-5}$ & $2.54 \cdot 10^{-5}$ \\
\hline 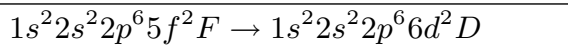 & $.04 \cdot 1$ & & \\
\hline $506-20$ & $3.85 \cdot 10^{-4}$ & & \\
\hline$s^{2} 2 s^{2} 2 p^{6} 5 f^{2} F_{5}$ & $.04 \cdot 10^{-4}$ & 4.42 & 4.43 \\
\hline $1 s^{2} 2 s^{2} 2 p^{6} 5 f^{2} F_{5 / 2} \rightarrow 1 s^{2} 2 s^{2} 2 p^{6} 6 d^{2} D_{5 / 2}$ & $.92 \cdot 10^{-5}$ & 2.108 & $2.11 \cdot 10^{-5}$ \\
\hline $7^{2} 2 a^{2} q^{6} \sigma^{2} P$ & $5.97 \cdot 10$ & -3 & \\
\hline${ }^{2} 2 s^{2} 2 p^{6} 6 p^{2} I$ & $97 \cdot 10^{-3}$ & -3 & $4.03 \cdot 10^{-3}$ \\
\hline $1 s^{2} 2 s^{2} 2 p^{6} 6 p^{2} P_{1 / 2} \rightarrow 1 s^{2} 2 s^{2} 2 p^{6} 7 s^{2} S_{1 / 2}$ & $.99 \cdot 10^{-3}$ & 2.01 & $2.02 \cdot 10^{-3}$ \\
\hline $1 s^{2} 2 s^{2} 2 n^{6} 6 n^{2} P$ & 64. & 2.24 & \\
\hline${ }^{2} 2 s^{2} 2 p^{6} 6 p^{2} P_{3}$ & $1.76 \cdot 10^{-3}$ & 1.492 & $1.50 \cdot 10^{-3}$ \\
\hline $1 s^{2} 2 s^{2} 2 p^{6} 6 p^{2} P_{1 / 2} \rightarrow 1 s^{2} 2 s^{2} 2 p^{6} 8 s^{2} S_{1 / 2}$ & $8.84 \cdot 10^{-4}$ & 7.5 & $7.52 \cdot 10^{-4}$ \\
\hline $1 s^{2} 2 s^{2} 2 p^{6} 6 d^{2} D$ & $1.21 \cdot 10^{-4}$ & 1.17 & $1.14 \cdot 10^{-4}$ \\
\hline $2 s^{2} 2 p^{6} 6 d$ & $1.09 \cdot 10^{-4}$ & )$^{-4}$ & 1.0 \\
\hline $2 s^{2} 2 p^{6} 6 d^{2} D_{3}$ & $1.19 \cdot 10^{-4}$ & 1.16 & $1.13 \cdot 10^{-4}$ \\
\hline $1 s^{2} 2 s^{2} 2 p^{6} 6 d^{2} D_{3 / 2} \rightarrow 1 s^{2} 2 s^{2} 2 p^{6} 7 p^{2} P_{3 / 2}$ & $1.21 \cdot 10^{-5}$ & $1.179 \cdot 10^{-5}$ & $1.15 \cdot 10^{-5}$ \\
\hline $1 s^{2} 2 s^{2} 2 p^{6} 6 d^{2} D \rightarrow 1 s^{2} 2 s^{2} 2 p^{6} 7 f^{2} F$ & $2.71 \cdot 10^{-3}$ & $2.550 \cdot 10^{-3}$ & $2.55 \cdot 10^{-3}$ \\
\hline${ }^{2} 2 s^{2} 2 p^{6} 6 d^{2} D_{5 / 2} \rightarrow 1$ & $2.71 \cdot 10^{-3}$ & $2.550 \cdot 10^{-3}$ & $2.55 \cdot 10^{-3}$ \\
\hline${ }^{2} 2 s^{2} 2 p^{6} 6 d^{2} D_{3 / 2} \rightarrow 1$ & $2.53 \cdot 10^{-3}$ & $2.38 \cdot 10^{-3}$ & $2.38 \cdot 10^{-3}$ \\
\hline $1 s^{2} 2 s^{2} 2 p^{6} 6 d^{2} D_{5 / 2} \rightarrow 1 s$ & $1.80 \cdot 10^{-4}$ & $1.700 \cdot 10^{-4}$ & $1.70 \cdot 10^{-4}$ \\
\hline $1 s^{2} 2 s^{2} 2 p^{6} 6 d^{2} D \rightarrow 1 s^{2} 2 s^{2} 2 p^{6} 8 f^{2} F$ & $1.54 \cdot 10^{-3}$ & $2.106 \cdot 10^{-3}$ & $1.95 \cdot 10^{-3}$ \\
\hline${ }^{2} 2 s^{2} 2 p^{6} 6 d^{2} D_{5 / 2} \rightarrow 1 s^{2} 2 s^{2} 2 p^{6} 8 f^{2} F_{7 / 2}$ & $1.54 \cdot 10^{-3}$ & $2.106 \cdot 10^{-3}$ & $1.95 \cdot 10^{-3}$ \\
\hline$s^{2} 2 s^{2} 2 p^{6} 6 d^{2} D_{3 / 2} \rightarrow 1 s$ & $1.43 \cdot 10^{-3}$ & $1.966 \cdot 10^{-3}$ & $1.82 \cdot 10^{-3}$ \\
\hline $1 s^{2} 2 s^{2} 2 p^{6} 6 d^{2} D_{5 / 2} \rightarrow 1 s$ & $1.02 \cdot 10^{-4}$ & $1.404 \cdot 10^{-4}$ & $1.30 \cdot 10^{-4}$ \\
\hline $1 s^{2} 2 s^{2} 2 p^{6} 7 p^{2} P$ & $2.65 \cdot 10^{-3}$ & $2.676 \cdot 10^{-3}$ & $2.67 \cdot 10^{-3}$ \\
\hline$s^{2} 2 s^{2} 2 p^{6} 7 p^{2} P_{3}$ & $1.76 \cdot 10^{-3}$ & $1.782 \cdot 10^{-3}$ & $1.78 \cdot 10^{-3}$ \\
\hline $1 s^{2} 2 s^{2} 2 p^{6} 7 p^{2} P_{1 / 2} \rightarrow 1 s^{2} 2 s^{2} 2 p^{6} 8 s^{2} S_{1 / 2}$ & $8.87 \cdot 10^{-4}$ & $8.94 \cdot 10^{-4}$ & $8.92 \cdot 10^{-4}$ \\
\hline
\end{tabular}


TABLE (cont.)

\begin{tabular}{l|c|c|c}
\hline \hline Transitions & $\begin{array}{c}\text { This } \\
\text { work }\end{array}$ & $\begin{array}{c}\text { MCHF } \\
\text { (Ref. [16]) }\end{array}$ & $\begin{array}{c}\text { Acc. val. } \\
(\text { Ref. [15]) }\end{array}$ \\
\hline $1 s^{2} 2 s^{2} 2 p^{6} 7 p^{2} P \rightarrow 1 s^{2} 2 s^{2} 2 p^{6} 9 s^{2} S$ & $1.18 \cdot 10^{-3}$ & $1.166 \cdot 10^{-3}$ & $1.09 \cdot 10^{-3}$ \\
$1 s^{2} 2 s^{2} 2 p^{6} 7 p^{2} P_{3 / 2} \rightarrow 1 s^{2} 2 s^{2} 2 p^{6} 9 s^{2} S_{1 / 2}$ & $7.87 \cdot 10^{-4}$ & $7.764 \cdot 10^{-4}$ & $7.25 \cdot 10^{-4}$ \\
$1 s^{2} 2 s^{2} 2 p^{6} 7 p^{2} P_{1 / 2} \rightarrow 1 s^{2} 2 s^{2} 2 p^{6} 9 s^{2} S_{1 / 2}$ & $3.95 \cdot 10^{-4}$ & $3.903 \cdot 10^{-4}$ & $3.64 \cdot 10^{-4}$ \\
\hline $1 s^{2} 2 s^{2} 2 p^{6} 8 p^{2} P \rightarrow 1 s^{2} 2 s^{2} 2 p^{6} 9 s^{2} S$ & $1.31 \cdot 10^{-3}$ & $1.604 \cdot 10^{-3}$ & $1.28 \cdot 10^{-3}$ \\
$1 s^{2} 2 s^{2} 2 p^{6} 8 p^{2} P_{3 / 2} \rightarrow 1 s^{2} 2 s^{2} 2 p^{6} 9 s^{2} S_{1 / 2}$ & $8.78 \cdot 10^{-4}$ & $1.068 \cdot 10^{-4}$ & $8.52 \cdot 10^{-4}$ \\
$1 s^{2} 2 s^{2} 2 p^{6} 8 p^{2} P_{1 / 2} \rightarrow 1 s^{2} 2 s^{2} 2 p^{6} 9 s^{2} S_{1 / 2}$ & $4.41 \cdot 10^{-4}$ & $5.359 \cdot 10^{-4}$ & $4.27 \cdot 10^{-4}$
\end{tabular}

very sensitive as $2-4 \%$. It can be seen from Table that our results being presented in this work for transition probabilities calculated by using WBEPM theory are in agreement with accepted values from NIST and MCHF results.

The both theoretical and experimental determination of transition probabilities for multi-electron systems is an important and difficult problem in atomic physics. While the calculation procedure for the systems with a few electrons can be carried out easily, the calculations become more difficult and complex in the case of increasing numbers of electrons. It is not easy to deal with these multi-electron systems using well known standard methods, since many configurations and orbital basis-set functions may be necessary to be taken into account to obtain accurate results for especially highly excited levels. Therefore, many of the used methods have considered only low lying levels which are very insufficient for practical and astrophysical applications. Many theoretical methods do not consider fine-structure effects and only provide multiplet results. Moreover, the most of theoretical and experimental data in the literature are generally restricted only to transitions involving low lying levels. The studies of physical parameters such as transition probabilities, oscillator strengths and lifetimes of alkali metal atoms, heavy metal atoms and many valence electron systems in the literature which have been carried out by Zheng et al. using WBEPM theory demonstrate the reliability of present method. The determination of the expectation values of the radii is very crucial in the WBEPM theory. Thus, to obtain more sensitive and reliable results, we have employed the numerical NRHF wave functions for the expectation values of radii in all relevant excited states of neutral sodium. Previously, we have employed NRHF wave functions for determination of relevant parameters in atomic nitrogen [19, 20], lithium [21], oxygen [22], fluorine [23], and potassium [24] and have obtained very satisfactory results in calculation of transition probabilities and oscillator strengths.

In this work, we also have obtained higher transition probabilities than those obtained in previous works for transitions between excited states. We have seen that by using numerical non-relativistic Hartree-Fock wave functions for expectation values of radii, the calculation of transition probabilities gives results in agreement with literature. The use of the WBEPM theory is advantageous for 
much more complicated systems, especially for those in highly lying states. According to the WBEPM theory, transition probability values will be better, if the expectation value of radius belonging to the levels is accurate enough.

\section{Acknowledgments}

The authors gratefully acknowledge the Selçuk University Scientific Research Projects (BAP) Coordinating Office for the financial support.

\section{References}

[1] J. Musielok, W.L. Wiese, G. Veres, Phys. Rev. A 51, 3588 (1995).

[2] H.P. Saha, C.F. Fischer, Phys. Rev. A 15, 1046 (1977).

[3] A. W. Weiss, Can. J. Chem. 70, 456 (1992).

[4] N.W. Zheng, Chin. Sci. Bull. 33, 916 (1988).

[5] N.W. Zheng, T. Wang, R.Y. Yang, Y.G. Wu, J. Chem. Phys. 112, 7042 (2000).

[6] N.W. Zheng, T. Wang, D.X. Ma, T. Zhou, J. Fan, Int. J. Quant. Chem. 98, 281 (2004).

[7] N.W. Zheng, T. Wang, Int. J. Quant. Chem. 98, 495 (2004).

[8] N.W. Zheng, T. Zhou, T. Wang, R.Y. Yang, Y.J. Sun, F. Wang, C.G. Chen, Phys. Rev. A 65, 052510 (2002).

[9] N.W. Zheng, A New Outline of Atomic Theory, Jiang Su Education Press, Nanjing, People's Republic of China 1988.

[10] N.W. Zheng, Chin. Sci. Bull. 22, 531 (1977).

[11] N.W. Zheng, T. Wang, T. Zhou, D.X. Ma, J. Phys. Soc. Jpn. 71, 1672 (2002).

[12] N.W. Zheng, J. Fan, D.X. Ma, T. Wang, J. Phys. Soc. Jpn. 72, 3091 (2003).

[13] R.D. Cowan, The Theory of Atomic Structure and Spectra, University of California Press, Berkeley 1981.

[14] G. Gaigalas, C.F. Fischer, Comput. Phys. Commun. 98, 255 (1996).

[15] A. Lindgard, S.E. Nielsen, At. Data Nucl. Data Tables 19, 533 (1977).

[16] Y. Ralchenko, F.C. Jou, D.E. Kelleher, A.E. Kramida, A. Musgrove, J. Reader, W.L. Wiese, K. Olsen, NIST Atomic Spectra Database (version 3.0.1) URL:http://physics.nist.gov, National Institute of Standards and Technology, Gaithersburg, MD 2006.

[17] W.L. Wiese, Atomic transition probabilities (Web Version 2.0): URL:http://physics.nist.gov/PhysRefData/datarefs/IAU/WG2/report2.html, National Institute of Standards and Technology, Gaithersburg, MD 2006.

[18] C.F. Fischer, the MCHF and MCDHF collection, Vanderbilt University, Nashville, TN http://www.vuse.vanderbilt.edu/ cff/mchf collection, 2002.

[19] G. Çelik, E. Akín, H.Ş. Kílíç, Eur. J. Phys. D 40, 325 (2006).

[20] G. Çelik, E. Akín, H.Ş. Kílíç, Int. J. Quant. Chem. 107, 495 (2007).

[21] G. Çelik, J. Quantum. Spectrosc. Radiat. Transfer 103, 578 (2007).

[22] G. Çelik, Ş. Ateş, Eur. J. Phys. D 44, 433 (2007).

[23] G. Çelik, H.Ş. Kílíç, E. Akn, Tur. J. Phys. 30, 165 (2006).

[24] G. Çelik, Ş. Ateş, to be published in Can. J. Phys. 